L'HOMME L'Homme

Revue française d'anthropologie

202 | 2012

Varia

\title{
Michel Izard (1931-2012)
}

Dominique Casajus et Fabio Viti

\section{(2) OpenEdition}

Journals

Édition électronique

URL : http://journals.openedition.org/lhomme/23011

DOI : 10.4000//homme.23011

ISSN : 1953-8103

Éditeur

Éditions de l'EHESS

Édition imprimée

Date de publication : 4 juin 2012

Pagination : 7-13

ISSN : 0439-4216

\section{Référence électronique}

Dominique Casajus et Fabio Viti, « Michel Izard (1931-2012)», L'Homme [En ligne], 202 | 2012, mis en ligne le 29 mai 2014, consulté le 14 septembre 2020. URL : http://journals.openedition.org/lhomme/ 23011

(c) École des hautes études en sciences sociales 


\title{
Michel Izard (|93|-20|2)
}

\author{
Dominique Casajus \& Fabio Viti
}

$C^{2}$

EST EN 1957 que Michel Izard a fait connaissance avec les hommes auxquels il allait vouer l'essentiel de son ouvre: les Moose du Yatenga, au nord-ouest du pays qui s'appelait la Haute-Volta (Burkina Faso actuel).

On envisageait alors d'établir dans les zones fertilisées par le Sourou, un affluent-défluent de la Volta Noire, des paysans moose installés jusque-là un peu plus à l'est, sur les terres ingrates du Yatenga. L'Institut des sciences humaines appliquées de l'Université de Bordeaux et le Service de l'hydraulique

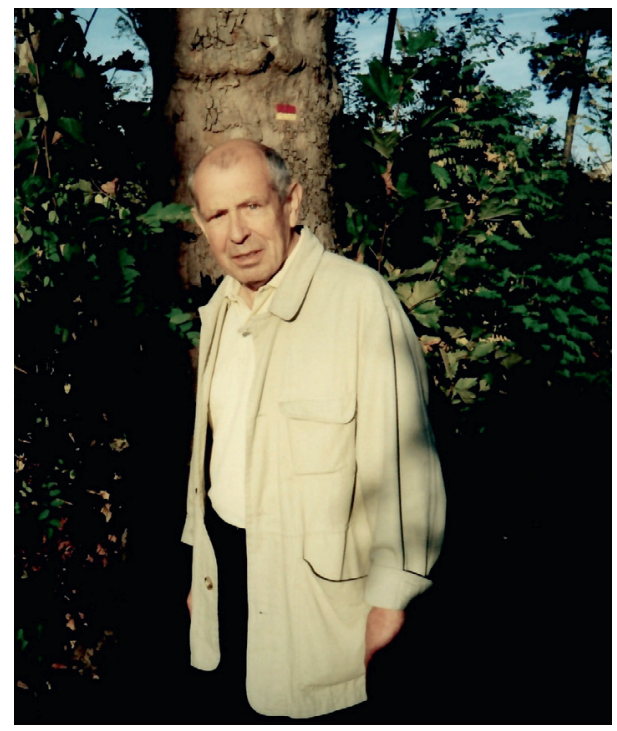

Michel Izard, Lubéron, 200 I (Cl. Marie Mauzé) de Ouagadougou l'avaient chargé d'évaluer l'impact du projet.

Il devait enquêter dans les villages moose d'où l'on comptait faire venir les colons, tandis que les enquêtes dans les villages samo et marka appelés à les accueillir étaient confiées à Françoise Héritier.

A cette carrière d'ethnologue dont il effectuait ainsi les tout premiers pas, ses études initiales ne semblaient pas l'avoir préparé. Au sortir du lycée Pasteur de Neuilly-sur-Seine, il sétait d'abord inscrit dans une classe de mathématiques supérieures qu'il avait abandonnée peu après pour

Une version plus ample de ce texte doit servir d'introduction à un volume d'hommages à Michel Izard, La Terre et le Pouvoir, que les éditions du CNRS feront paraître en juin de cette année. 
une propédeutique de lettres, avant de sorienter vers la philosophie. Là, il avait bénéficié de l'enseignement de Jean Hippolyte, Jean Wahl, Vladimir Jankélévitch, Ferdinand Alquié... Son mémoire de diplôme d'études supérieures (DES), rédigé en 1956 sous la direction de Jean Wahl, portait le titre Négation et négativité chez Spinoza, Hegel et dans la philosophie de Dom Deschamps. La règle voulait que les agrégatifs de philosophie passent un certificat "scientifique", et beaucoup parmi eux optaient pour le certificat d'ethnologie délivré par l'Institut d'ethnologie du Musée de l'Homme. Il fut donc amené à suivre là des enseignements qui - il l'a confié dans un de ses derniers entretiens ${ }^{1}-$ n'avaient assurément pas l'envergure de ceux que lui dispensèrent ses maîtres en philosophie. Il décida cependant de se consacrer à l'ethnologie, et se mit à suivre les séminaires de l'École pratique des hautes études, où sa préférence allait aux chercheurs et enseignants américanistes: Claude Lévi-Strauss, Jacques Soustelle, Guy Stresser-Péan, Roger Bastide. C'est ainsi qu'il se souvenait d'avoir planché durant trois heures sur l'analyse componentielle de la terminologie de parenté chez les Indiens Pawnee selon Ward H. Goodenough...

Au moment où il arrive au Yatenga, il ne connaît à peu près rien de l'Afrique, et a dîu se documenter en hâte, entre autres chez Louis Tauxier. Le Yatenga est le second par l'importance territoriale et démographique de la vingtaine de royaumes qui constituaient à la fin du XIX siècle le Moogo, ou pays des Moose ${ }^{2}$. Michel Izard l'aura parcouru durant près de vingt ans, dans une enquête conduite village par village, quartier par quartier. Au total, près de 2200 quartiers qu'il répertorie systématiquement, et où il recueille traditions orales, récits de fondation, histoire du peuplement, chronologie des règnes et des commandements, bref, la trame sur laquelle viendra se fonder une réflexion anthropologique, toujours soucieuse de dialoguer avec l'histoire. L'entreprise aboutit, en 1980, à la soutenance d'une thèse d'État intitulée Les Archives orales d'un royaume africain. Recherches sur la formation du Yatenga, dont une partie a été publiée en 1985 sous la forme de deux ouvrages: Gens du pouvoir, gens de la terre. Les institutions politiques de l'ancien royaume du Yatenga (Bassin de la Haute-Volta Blanche) et Le Yatenga précolonial. Un ancien royaume du Burkina, qui retrace l'histoire du royaume depuis le XVI siècle - date supposée de l'arrivée des conquérants moose - jusqu'à la fin du XIX siècle. Plus tard viendront: L'Odyssée du pouvoir. Un royaume africain : État, société, destin individuel (1992)33, et enfin Moogo. L'émergence d'un espace étatique ouest-africain au $\mathrm{XVI}^{\mathrm{e}}$ siècle. Étude d'anthropologie historique (2003).

1. Cf. Michel Izard, L'Ethnologie en héritage, Paris, La Huit production, 2008. Sauf mention contraire, les propos de Michel Izard cités dans la suite proviennent de cet entretien.

2. Moose est, dans la transcription devenue usuelle, la forme plurielle du nom de ceux que les vieux auteurs appellent plutôt les Mossi. Le singulier est Moaga. 
Dialoguer avec l'histoire, avons-nous dit. De fait, Michel Izard reconnaissait dans l'entretien déjà cité avoir "plus d'intérêt pour l'histoire que pour l'anthropologie des mythes, des rituels, etc. (tout en ayant un grand respect pour ce genre de démarche)". À quoi il ajoutait avoir "la naïveté de croire que l'histoire, ça aide directement les gens chez lesquels on a travaillè". Ainsi, dans le dossier qu'il présenta en 1986, à l'appui d'une demande de promotion au grade de directeur de recherches de classe exceptionnelle du Centre national de la recherche scientifique, il écrivait à propos de son Yatenga précolonial :

"Écrit de sorte qu'il soit accessible à des non-spécialistes, il est certainement, de tous les travaux que j'ai publiés, celui dont la rédaction et ce qu'il est convenu d'appeler la "réception" m’ont procuré le plus de plaisir, simplement parce que je le destinais aux gens du Yatenga et que ceux-ci, après que j'ai pu en assurer une très large diffusion parmi eux, en ont fait leur bien propre: beau témoignage de satisfaction pour celui dont, à son corps défendant, ils avaient fait leur rogemmiki naaba, créant à cette occasion une fonction dont l'objet était la conservation de ce que l'on est "né trouver". Même si je me suis parfois inquiété de voir promues au rang de "faits" ce qui n'est pour l'historien que fragiles constructions à la valeur de vérité souvent indécidable [...], j’ai trouvé, dans l'accueil fait à ce que l'on appelle lourdement aujourd'hui la restitution de l'information, l'écho du souci de mes informateurs des années 19631971 de savoir si ce qu'ils me disaient se retrouverait bien dans "le livre" que je n'allais certainement pas manquer d'écrire en manière d'hommage à leur sourcilleuse passion pour la gloire du "pays de Naaba Yaadga" [le fondateur du royaume]».

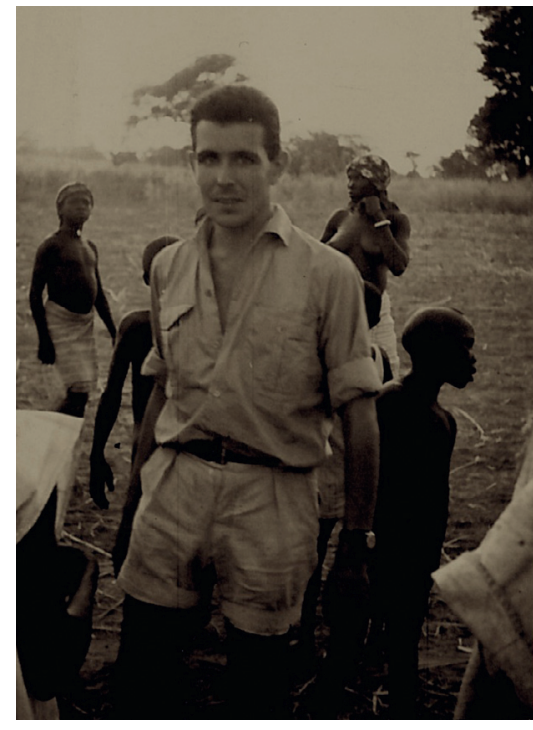

Michel Izard, Haute-Volta, vers 1960

Il suffit cependant de lire une page de Michel Izard pour l'identifier sans hésiter comme un anthropologue. En même temps quil s'attachait à rendre le détail de la succession des événements, à en reconstituer la chronologie probable, il a voulu dégager la forme des phénomènes dont le pays moaga a été le théatre durant plus de quatre siècles. Forme dont, de sa thèse à ses derniers écrits, il s'est efforcé de circonscrire les traits les plus essentiels, parvenant à lui donner, au terme d'une longue distillation, la simplicité et la clarté d'une épure. Ce souci de réduire à l'essentiel les données recueillies, on le retrouverait certes chez des historiens, mais il a surtout caractérisé, en France du moins, l'anthropologie sociale.

3. Voir l'important À Propos que lui a consacré Emmanuel Terray, «L'État contre le pouvoir» (L'Homme, 1996, 138 : 121-126), texte que Michel Izard avait particulièrement apprécié et qui s'inscrivait dans un débat que tous deux n'ont cessé de nourrir et d'enrichir depuis leur rencontre à la fin des années 1960 et la longue amitié qui s’en est suivie. 
Les figures dont il a tracé les contours, en les dépouillant de l'accessoire, sont devenues le bien commun de la profession, la plus marquante - sa mise au jour restera un apport majeur - étant le face à face entre Gens du pouvoir et Gens de la terre: ces maîtres venus d'ailleurs, contraints de s'en remettre à leurs sujets autochtones du soin de propitier une terre nourricière sur laquelle la conquête ne leur a donné aucun pouvoir, quel enseignement d'anthropologie pourrait aujourd'hui se dispenser de les évoquer? De même pour ce roi auquel ses serviteurs, gens de basse extraction, ont peu à peu arraché la réalité d'un pouvoir dont il ne conserve que le faste et les pompes; pour les nakombse, ces hobereaux qui, leurs liens généalogiques avec le lignage royal seétant distendus au fil des générations, ne sont plus que des guerriers sans guerre et des seigneurs sans fief, riches de leur seul orgueil et tenaillés par leur amertume. Figures dont la contemplation nous ramène à des chemins maintes fois parcourus par les penseurs du politique. Si l'anthropologie consiste à rendre disponibles à la comparaison les matériaux singuliers qu'elle recueille dans les sociétés les plus diverses, alors l'euvre de Michel Izard est de part en part anthropologique.

Dans tout cela, l'élaboration théorique aura été inséparable du souci de l'écriture. Ses proches, tous ceux dont il a relu, annoté et souvent corrigé les manuscrits, ceux qui ont reçu de lui ces lettres dactylographiées où la relation de faits même ténus faisait l'objet d'un labeur scripturaire, savent son exigence dans ce domaine. Tout comme ils savent l'ancienneté et l'intimité de son commerce avec la littérature. En premier lieu les surréalistes, dont le contact remonte à ses années de lycée. Michel Izard le datait de l'exposition que la galerie Maeght leur a consacrée en 1947, alors qu'il n'avait que seize ans. S'il prit par la suite ses distances avec le mouvement, il a toujours "gardé un profond attachement au bouleversement du monde et de la sensibilité que [lui] a apporté le Surréalisme". Julien Gracq a aussi compté pour lui, et plus tard Walter Benjamin, dont il aborda l'euvre vers 1970.

Son souci de l'écriture, peut-être justement parce que sa longue fréquentation de quelques grands auteurs l'avait amené depuis longtemps à se défaire des naïvetés et clichés, a peu à voir avec la nombriliste complaisance où s'égare toute une ethnographie récente ou moins récente. Sobre et compacte, adamantine et ascétique, sa prose peut paraître froide, distanciée, "janséniste"; elle est surtout d'un auteur qui se méfiait de l'emphase et de l'effusion. Curieuse rencontre avec la société moaga elle-même, dont il a aimé "l'équilibre, l'absence de propension à l'excès". Le plaisir de l'écriture réside ici dans la soustraction, non dans l'expansion. On pense aux pages où, au début de son Sade, Fourier, Loyola, Roland Barthes rappelle que le style n'est pas un ajout à la pensée, mais la pensée elle-même, advenue dans l'écriture ${ }^{4}$. On peut aussi

4. Roland Barthes, Sade, Fourier, Loyola, Paris, Le Seuil, 1970. 
évoquer ce conseil d'écriture sur lequel Michel Volkovitch conclut son Verbier : "Le style: ce qui reste quand on a tout raboté" ".

Michel Izard aura beaucoup raboté. Le travail de la varlope se perçoit dans Le Yatenga précolonial..., livre quion pourrait dire narratif, mais où la densité et le souci de la synthèse annoncent déjà le tour si aphoristique des textes plus "théoriques". Il est poussé à l'extrême dans L'Odyssée du pouvoir..., recueil d'articles soumis pour cette édition à un impitoyable assèchement; là, rien n'a été gardé que l'essentiel: l'État, la société, la guerre, le destin individuel, l'identité, le sacrifice, le rapport à la terre et au territoire, les ancêtres, autant de sujets sur lesquels Michel Izard aura, de son écriture acérée, laissé une indélébile empreinte.

À côté de l'ouvre concernant les Moose, Michel Izard a dirigé ou codirigé une série d'ouvrages d'une portée plus générale: La Fonction symbolique. Essais d'anthropologie en 1979, avec Pierre Smith, livre qui donne une bonne idée d'ensemble de ce qu'étaient alors les réflexions de style structuraliste sur les mythes et le rituel; Antropologia delle tradizioni intellettuali: Francia e Italia en 2000, avec Fabio Viti; en 2004, un cahier de L'Herne consacré à Claude Lévi-Strauss. Et aussi le Dictionnaire de l'ethnologie et de l'anthropologie, publié en 1991 et qui depuis a fait l'objet de plusieurs rééditions et traductions. Entreprise dont il fut le principal maître d'ouvre, ce dictionnaire était le fruit d'un long chantier ouvert dès 1984 et autour duquel il a rassemblé la plupart des anthropologues français. À quoi sajoutent - témoignage de l'étendue de ses curiosités - toutes sortes d'articles, notes ou recensions qui ont pu porter aussi bien sur les sociétés africaines que sur la parenté bretonne, l'ethnologie nord-américaine, la figure de Volney ou le parcours de Walter Benjamin.

Tout comme son cuvre écrite, les responsabilités scientifiques et administratives quill a assumées dès le début de sa carrière auront concerné l'anthropologie en général autant que la recherche africaniste. Ses fonctions se sont notamment exercées au Centre voltaïque de la recherche scientifique (CVRS), comme directeur de 1968 à 1969 puis conseiller scientifique jusqu'en 1979; à l'ORSTOM (actuel IRD) où il a effectué diverses missions d'évaluation; au Centre national de la recherche scientifique où il a été, en 1982, chargé de mission auprès du Département des sciences de l'homme et de la société, et où il a présidé de 1983 à 1986 la section 33 ("Anthropologie, ethnologie, préhistoire ") du Comité national de la recherche scientifique. C'est d'ailleurs sur sa proposition que, lors du remodelage du Comité national en 1992, une nouvelle section spécifiquement consacrée à l'ethnologie a été baptisée "Unité de l'homme et diversité des cultures" : un intitulé qui pourrait aussi bien servir

5. Michel Volkovitch, Verbier. Herbier verbal, Paris, Nadeau, 2000. 
d'épigraphe à son auvre. On doit aussi mentionner les missions assurées pour le compte de l'UNESCO, du festival panafricain du cinéma de Ouagadougou (FESPACO), du Ministère de la recherche et de l'industrie, du Ministère de la coopération et du développement; les revues ou collections quil a animées ou auxquelles il a collaboré activement (Notes et documents voltaïques, Voix voltaïque, Systèmes de pensée en Afrique noire, Journal des africanistes, L'Homme, les "Cahiers de Gradhiva»); les enseignements qu'il a dispensés, les travaux quill a dirigés ou les séminaires qu'il a coordonnés, à l'École des hautes études en sciences sociales, à l'Université de Nanterre-Paris X, à l'Université de Ouagadougou. Comment ne pas évoquer, en particulier, le séminaire "Anthropologie sociale comparée", qu’il a organisé à l'EHESS, depuis le début des années 1980 jusqu'au milieu des années 1990. De grands noms sont venus s'y exprimer (Emmanuel Terray, Jean Pouillon, Denise Paulme, Alfred Adler, Claude Tardits, Raymond Firth, André Green et bien d'autres), et les débutants que nous étions alors, anthropologues de la génération suivante, ont presque tous eu l'occasion d'y faire leurs premières armes un jour ou l'autre. Dans tout cela, il aura été au service de la cité scientifique. Ses pairs, notamment ceux qui ont dirigé avec lui des ouvrages collectifs, savent quelle était sa disponibilité au dialogue et à l'accueil; ses cadets le savent plus encore, eux justement qu'il a accueillis en si grand nombre dans son séminaire, et qui étaient tellement surpris, lorsquils avaient franchi la porte de son bureau, de la générosité avec laquelle il leur prodiguait ses conseils. Souvent consacrées aux premiers ouvrages de jeunes collègues, ses recensions témoignent de l'intérêt qu'il leur portait : son exigence de rigueur n'allait pas sans bienveillance.

Tel fut l'homme de science qui vient de nous quitter. Mais, pour nous, Michel Izard était d'abord un ami. Nous l'admirions, nous l'aimions, et aujourd'hui nous l'avons perdu. Michel, resquiescas in pace.

Centre national de la recherche scientifique Centre d'études des mondes africains, Ivry-sur-Seine casajus@ivry.cnrs.fr

Università di Modena e Reggio Emilia Laboratorio di Etnologia, Modena (Italie) viti.fabio@libero.it 


\section{Izard, Michel}

1985a Gens du pouvoir, gens de la terre.

Les Institutions politiques de l'ancien royaume du Yatenga (bassin de la Haute-Volta Blanche). Paris, Éd. de la Maison des sciences de l'Homme/Cambridge, Cambridge University Press.

1985b Le Yatenga précolonial. Un ancien royaume du Burkina. Paris, Karthala.

1992 L'Odyssée du pouvoir. Un royaume africain: Etat, société, destin individuel. Paris, Éd. de l'École des hautes études en sciences sociales.

2003 Moogo. L'émergence d'un espace étatique ouest-africain au XVI siècle. Études d'anthropologie historique.

Paris, Karthala.
Izard, Michel, ed.

2004 Claude Lévi-Strauss.

Paris, Éd. de l'Herne.

Izard, Michel \& Pierre Bonte, eds

1991 Dictionnaire de l'ethnologie

et de l'anthropologie.

Paris, Presses universitaires de France.

Izard, Michel \& Pierre Smith, eds

1979 La Fonction symbolique. Essais

d'anthropologie. Paris, Gallimard

(«Bibliothèque des sciences humaines»).

Izard, Michel \& Fabio Viti

2000 Antropologia delle tradizioni

intellettuali. Francia e Italia.

Roma, CISU.

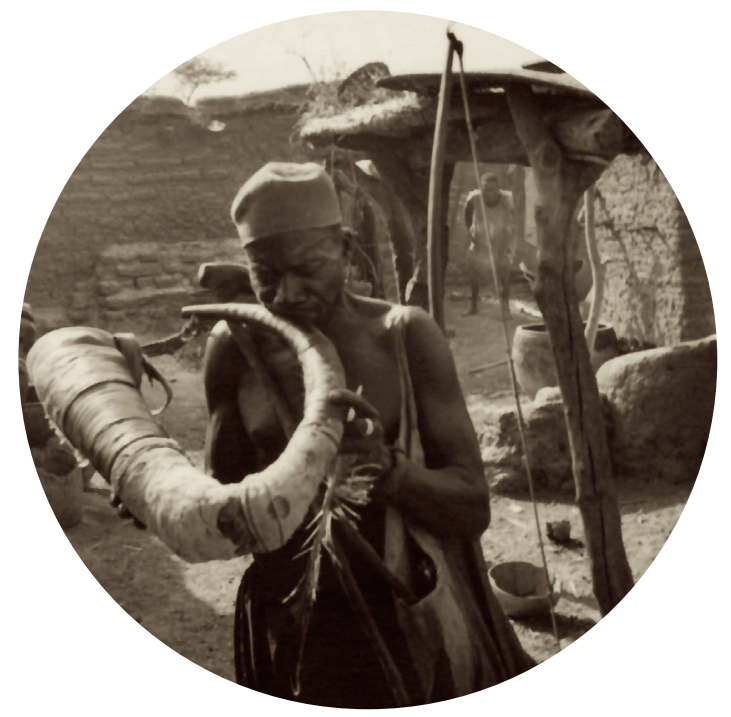

Les photographies présentées depuis cette page ont été prises par Michel Izard, lors de ses premiers terrains en Haute-Volta, en pays mossi (1957-1958, 1960-1963). 
